INPLASY

PROTOCOL

To cite: Chen et al. The relationship between BTLA rs1982809 polymorphism and the susceptibility of cancers: a meta-analysis. Inplasy protocol 202130023. doi:

10.37766/inplasy2021.3.0023

Received: 07 March 2021

Published: 08 March 2021

Corresponding author:

Yingliang Li

drliyingliang1977@163.com

Author Affiliation:

General Surgery Department, First Affiliated Hospital of

Nanchang University

Support: There was no

external funding.

Review Stage at time of this submission: Preliminary

searches.

Conflicts of interest:

None declared.

\section{The relationship between BTLA rs1982809 polymorphism and the susceptibility of cancers: a meta-analysis}

Chen, J1; Liu, R²; Wang, J3; Xiong, H4 Li, Y55.

Review question / Objective: As the primary cause of morbidity and mortality around the world and in every region of the world, cancers place a tremendous burden on the global medical systems, regardless of the level of human development. Hereditary factors probably play a key role in the genesis and progression of cancers. Polymorphism in BTLA gene is considered to be a risk factor for cancer. Currently, a few SNPs in BTLA were reported to be associated with cancer risks in some publications, BTLA rs1982809 polymorphism is common one of them. Several recent researches have probed the connection between the SNP of rs1982809 and cancer susceptibility and yielded different results, therefore, we conducted a meta-analysis to comprehensively access the relationship between BTLA rs1982809 polymorphism and tumor susceptibility.

Condition being studied: The connection between BTLA rs1982809 polymorphism and tumor risk has been investigated by several studies and yielded different results.

INPLASY registration number: This protocol was registered with the International Platform of Registered Systematic Review and Meta-Analysis Protocols (INPLASY) on 08 March 2021 and was last updated on 08 March 2021 (registration number INPLASY202130023).

\section{INTRODUCTION}

Review question / Objective: As the primary cause of morbidity and mortality around the world and in every region of the world, cancers place a tremendous burden on the global medical systems, regardless of the level of human development. Hereditary factors probably play a key role in the genesis and progression of cancers. Polymorphism in BTLA gene is considered to be a risk factor for cancer. Currently, a few SNPs in BTLA were reported to be associated with cancer risks in some 
publications, BTLA rs1982809 polymorphism is common one of them. Several recent researches have probed the connection between the SNP of rs1982809 and cancer susceptibility and yielded different results, therefore, we conducted a meta-analysis to comprehensively access the relationship between BTLA rs1982809 polymorphism and tumor susceptibility.

Condition being studied: The connection between BTLA rs1982809 polymorphism and tumor risk has been investigated by several studies and yielded different results.

\section{METHODS}

Search strategy: Eligible researches incorporated in this meta-analysis were screened and identified in five online literature data banks (PubMed, Web of Science, Embase, Wan Fang and CNKI) by two independent investigators. The keywords we utilized were: "B and $T$ lymphocyte attenuator or BTLA or LOC112268446 or rs1982809 or rs386551325 or rs60386396" and "neoplasm or tumor or cancer or carcinoma" and "polymorphism or SNP or allele or variation".

Participant or population: Case group and control group were extracted from previous case-control studies on the relationship between BTLA rs1982809 polymorphism and tumors.

Intervention: Patients of different races with tumors.

Comparator: Healthy people.

Study designs to be included: Case-control studies, tumor patients were included in the case group and healthy people were included in the control group.

Eligibility criteria: Inclusion criteria:(a) the studies investigated the connection between BTLA rs1982809 polymorphism and tumor risks; (b) the studies were casecontrol studies, tumor patients were included in the case group and healthy people were included in the control group; (c) the studies provided detail genotype frequencies. Exclusion criteria:(a) nonhuman trials; (b) duplicate articles.

Information sources: Eligible researches incorporated in this meta-analysis were screened and identified in five online literature data banks (PubMed, Web of Science, Embase, Wan Fang and CNKI) by two independent investigators.

Main outcome(s): The relationship between BTLA rs1982809 polymorphism and the susceptibility of cancers.

Additional outcome(s): The assication of BTLA rs1982809 polymorphism with tumors in people of different races and different types of tumors.

Quality assessment / Risk of bias analysis: Sensitivity analysis was performed excluding studies whose allele frequencies in controls exhibited significant deviation from the Hardy-Weinberg Equilibrium (HWE), given that the deviation may denote bias. Moreover, the extent to which the combined risk estimate might be affected by individual studies was assessed consecutively omitting every study from the meta-analysis (leave-one-out sensitivity analysis). Begg's funnel plots and Egger's linear regression test were used to investigate the publication bias.

Strategy of data synthesis: Pooled ORs and $95 \% \mathrm{Cl}$ were utilized to access the relation between BTLA rs1982809 polymorphism and tumor susceptibility in the dominant, recessive, homozygous, heterozygous and additive models. ORs were merged via the random or fixed effects model, and the selection of models depends on the heterogeneity of studies. We used the $Q$ and $I^{2}$ tests to evaluate the heterogeneity of genetic models. The random-effects model was applied when the heterogeneity exists in available studies(P50\%); oppositely, the fixed-effects model was utilized.

Subgroup analysis: Subgroup analysis based on the ethnicity, cancer-type and control source were conducted. 
Sensitivity analysis: Sensitivity analysis was applied to evaluate stability of the results via the leave-one-out method.

Country(ies) involved: China.

Ke ywords: single-nucleotide polymorphisms, rs1982809, meta-analysis, BTLA, cancer susceptibility.

Contributions of each author:

Author 1 - Jian Chen - Responsible for the conception and design of the project.

Author 2 - Ruihao Liu - Responsible for extracting data.

Author 3 - Jun Wang - Responsible for data analysis.

Author 4 - Haiwei Xiong - Responsible for literature search.

Author 5 - Yingliang Li - Corresponding author.

Email: drliyingliang1977@163.com 